\title{
NUEVOS ENFOQUES DE ÉTICA Y DEONTOLOGÍA APLICADOS A LA FORMACIÓN PROFESIONAL DEL CONTADOR PÚBLICO
}

\author{
NEW APPROACHES OF ETHICS AND DEONTOLOGY APPLIED TO THE \\ PROFESSIONAL FORMATION OF THE PUBLIC ACCOUNTANT
}

\author{
Ernesto A. Polar Falcón ${ }^{1}$ \\ Docente Principal de la Facultad de Ciencias Contables, UNMSM
}

[Recepción: Setiembre de 2010 / Conformidad: Octubre de 2010]

\section{RESUMEN}

El presente artículo muestra los avances en el proceso de investigación relacionado con el título de "NUEVOS ENFOQUES DE ÉTICA Y DEONTOLOGÍA APLICADOS A LA FORMACIÓN PROFESIONAL DEL CONTADOR PÚBLICO”. Se inicia informando el procesos de evaluación de las Propuestas de Estudio de Investigación CON CON - 2010 y la calificación con el más alto puntaje, en mérito a la importancia del tema para lograr una mejor formación del Contador Público en el Perú y a la metodología aplicada que se propuso. Ofrece los mensajes de dos interesantes Trabajos Interamericanos de la XXVIII CIC - 2009 y de un artículo de la Revista CONTADURÍA PÚBLICA, con enfoques distintos pero dirigidos al tema de la Ética y la Deontología Profesional y que sirven para cumplir con el primer objetivo del estudio de investigación propuesto, que puede aumentar sus fuentes de información en base al desarrollo de los dos objetivos adicionales, actualmente ya terminados y que se presentarán oportunamente. Lo importante, es crear conciencia sobre la necesidad de mejorar la calidad ética del Contador Público, preparando a los estudiantes para el desarrollo futuro de sus actividades con una personalidad enmarcada en los principios éticos que se plasman en la Deontología Profesional, para su reconocimiento en la socie-

\begin{abstract}
EThe article present progress shows progress process related to the title "NEW APPROACHES OF ETHICS AND DEONTOLOGY APPLIED TO THE PROFESSIONAL FORMATION OF THE PUBLIC ACCOUNTANT”. It begins by informing the assessment process of Proposed Research Study WITH CON 2010 and the rating with the highest score, in recognition of the importance of the issue to get a better practitioner training in Peru and applied methodology proposed. Offers two interesting posts of the XXVIII Interamerican Jobs CIC - 2009 and an article in the journal Public Accounting, but led to different approaches to the subject of Ethics and Professional Ethics and serve to meet the first objective of the study proposed research, which can increase their sources of information based on the development of two additional targets, now finished and will be presented in due course. The important thing is to create awareness about the need to improve the ethical quality of a public accountant, preparing students for the future development of its activities with a personality framed by ethical principles that are upheld by the Professional Ethics for recognition in Peruvian society, which they render professional services. The entire contents of the two American works and
\end{abstract}

* Doctor en Educación, UNMSM. Magíster en Contabilidad. Contador Público Colegiado Certificado. Docente Principal de la Facultad de Ciencias Contables UNMSM. E-mail: epolarf@hotmail.com 
dad peruana, donde prestarán sus servicios profesionales. Los contenidos completos de los dos Trabajos Interamericana y de la revista profesional, deben ser difundidos por el Instituto de Investigación de la Facultad de Ciencias Contables de la UNMSM, para motivar a su aplicación en la labor docente diaria.

Palabras clave: Ética y deontología profesional, estudios de investigación, nuevos enfoques de ética.

\section{INTRODUCCIÓN}

La Propuesta de Estudio de Investigación 2010, titulada: "NUEVOS ENFOQUES DE ÉTICA Y DEONTOLOGÍA APLICADOS A LA FORMACIÓN PROFESIONAL DEL CONTADOR PÚBLICO” fue presentada en diciembre de 2009, conjuntamente con otras Propuestas de Estudio de Investigación 2010 de la Facultad de Ciencias Contables de la UNMSM, al Comité de Evaluación de Estudios de Investigación CON CON 2010, formado por tres prestigiosos Catedráticos de otras Facultades de la UNMSM, que aceptaron evaluarlas por encargo del Vicerrectorado de Investigación, marcando como premisas, sus elevados niveles académicos, sus experiencias en el campo de la investigación y la imparcialidad absoluta en el trabajo de evaluación, por no estar vinculados a las actividades académicas de la Facultad de Ciencias Contables.

Los resultados de la mencionada evaluación, dio como resultado que la presente Propuesta relacionada con los temas de Ética y Deontología, alcanzara el mayor puntaje de calificación, tanto en su estructura metodológica como en su contenido temático. Los Catedráticos Evaluadores son conscientes de la enorme importancia que tiene en sus Facultades, la enseñanza de Ética y Deontología en la formación de profesionales que egresen capacitados para desarrollar sus ac- the professional journal, should be released by the Research Institute of the School of Accounting Sciences of San Marcos, to encourage their application in everyday teaching.

Key words: Ethics and professional deontology, research studies, new approaches of ethics.

tividades en un marco de pleno conocimiento de la puesta y cumplimiento práctico de la Ética como valor central y la Deontología como conjunto ordenado de deberes y obligaciones morales que se aplican en una determinada materia.

Se propuso como tema de investigación, los temas de Ética y Deontología, por estar plenamente convencido de la importancia que tienen para la Facultad de Ciencias Contables, en la formación profesional del Contador Público, quien en sus actividades futuras estará constantemente expuesto a cometer infracciones ya enunciadas en el Código de Ética Profesional vigente y otras que todavía no se han incluido como tales.

En la Propuesta de Estudio de Investigación, se anota como primer objetivo de trabajo: "Estudiar los contenidos de nuevos enfoques de Ética y Deontología, así como de formación del Contador Público, de la XXVIII Conferencia Interamericana de Contabilidad -CIC 2009 y otros, para adaptación curricular".

Existe plena justificación para el desarrollo de este Trabajo de Investigación, por varias razones: 1) Afianzar las bases académicas y profesionales que sustentaron la acreditación lograda por la Facultad de Ciencias Contables de la UNMSM en el año 2009; 2) Los estudios limitados de Ética y Deontología que actualmente se incluyen en el curso de Filosofía en breves capítulos, en el vigente 
Plan Curricular, que no despiertan interés en los alumnos ingresantes al Primer Semestre Académico; 3) El estudio de nuevos enfoques de Ética y Deontología, para incentivar a la creación de un nuevo curso de ÉTICA Y DEONTOLOGÍA PROFESIONAL, que reforzará el área de Humanidades; y 4) Por cuanto estos temas, se ubican en los permanente estudios de Ética y Ejercicio Profesional, así como de Educación del Instituto de Investigación de la Facultad de Ciencias Contables de la UNMSM y de los trabajos interamericanos y nacionales de las Conferencias Interamericanas de Contabilidad.

\section{ÁREAS DE LOS NUEVOS ENFOQUES DE ÉTICA Y DEONTOLOGÍA - CONFERENCIA INTERAMERICANA DE CONTABILIDAD}

En el Área 6. Comisión de Ética y Ejercicio Profesional de la Conferencia Interamericana de Contabilidad - CIC 2009, está el Trabajo Interamericano titulado: "DE LA TRANSDISCIPLINARIEDAD DE LA ÉTICA A LOS CÓDIGOS DEONTOLÓGICOS”, presentado por la colega R. Bianca Emmi - República Oriental de Uruguay, con extenso e interesante contenido, debidamente estructurado con los siguientes subtítulos:

- Resumen

- Introducción

- Precisiones Conceptuales

- El Papel de la Ética en una Moralidad Fracturada

- La Ética del Discurso

- Los Niveles de Reflexión Ética

- Características de la Ética Aplicada

En el Subtítulo de "RESUMEN", con la exposición breve de los aspectos más impor- tantes de su trabajo, es interesante transcribir el contenido de los dos párrafos, en el primero afirma que:

\section{SEGUNDO PÁRRAFO}

De los diferentes conceptos que se procuran precisar, lo más relevante lo constituye la diferencia entre la denominada Ética Profesional y la Deontología Profesional. La Ética hace directamente referencia a la conciencia personal autónoma y está orientada al bien, a la trascendencia del profesional como ser humano. La Deontología, en cambio, si bien se refiere al ámbito de acción libre de la persona, expresa aquellas obligaciones prácticas referidas a la moralidad, que se suponen consensuadas en el colectivo, y que carecen de control por parte de la legislación pública. Gravitan en la Deontología, el grado de compromiso y conciencia moral referidos a la profesión. Mediante la utilización de distintos instrumentos como códigos, libros de estilo, estatutos de redacción, convenios, manuales de buenas prácticas entre otros, la Deontología procura que un colectivo determine sus propios límites y se comprometa a estudiarlos $y$ no transgredirlos.

Así mismo, continúa:

\section{CUARTO PÁRRAFO}

"En el ámbito de la Ética Profesional gravita la conciencia moral y es imposible lograr una dignidad profesional si no se educa para resolver problemas complejos teniendo como faro el interés público y el bien común. La educación en Ética a nivel universitario debe ser explí- 
cita para generar reflexión y orientación en la resolución de dilemas morales".

En el Área 4. Comisión de Educación, está el Trabajo Interamericano titulado: "LA FORMACIÓN DEL CONTADOR PÚBLICO - UNA PROPUESTA PARA LA CARRERA DE GRADO", presentado por los colegas Ricardo I. M. Pahlen Acuña y Ana María Campo, de la República Argentina, precisando una moderna propuesta para la formación del Contador Público. De los ocho párrafos del Sub Título de "RESUMEN", se transcribe el siguiente:

Ante la disyuntiva de una formación generalista o especializada, entendemos que resulta necesario en primer lugar establecer un título habilitante de la profesión para cuya obtención se requieran estudios universitarios con una duración normal de cinco años. En lo inherente a la formación universitaria del Contador Público se considera que el profesional debe ser conocedor del medio que rodea el desenvolvimiento de las actividades económicas y jurídicas, única circunstancia que ha de otorgarle la posibilidad de expresarse en cualquier oportunidad, a través del lenguaje contable correspondiente, cumplimentando cualquier requerimiento que le presente la sociedad con conocimientos técnicos científicos apropiados y adecuada complementación humanística.

En esa "adecuada complementación humanística” se encierra sin lugar a dudas los conocimientos de Ética y Deontología Profesional, conjuntamente con los "conocimientos técnicos científicos apropiados", que sustentan la esencia de este Trabajo Interamericano.
En la Revista "CONTADURÍA PÚBLICA” - XXVIII CONFERENCIA INTERAMERICANA DE CONTABILIDAD - CANCÚN 2009, se publica el artículo titulado: "ÉTICA, NORMATIVA Y CALIDAD", de los colegas Herómines Enrique Montero Morán y José Rafael Zaá Méndez, de la República de Venezuela y vinculados con la Asociación Interamericana de Contabilidad - AIC. En sus cinco primeros párrafos se precisa lo siguiente:

La búsqueda de estándares de mayor calidad en el ejercicio profesional es, por lo tanto, un propósito a cuyo logro cooperan las estructuras fundantes de la profesión, las reglas que norman el ejercicio profesional y los principios éticos presentes en la conducta profesional.. Esto adquiere mayor relevancia cuando se trata del ejercicio de profesiones globalizadas, cuyo desarrollo transciende las fronteras de las naciones $y$ tiende a regirse por un lenguaje común, para unificar normas y procedimientos cuando la complejidad de los asuntos profesionales así lo requieren. Este es el caso de la Contaduría Pública, la cual ante las crisis y dilemas sufridos tiende a levantarse sobre objetivos de mayor control de calidad profesional y sobre la base de una ética fundada en la práctica de la normativa contable.

Lo anterior, quiere decir que para llegar a niveles de mayor calidad en el ejercicio profesional, se debe transitar por el camino de la aplicación correcta de la normativa contable como expresión praxeológica de una ética científica fundada en la experiencia.

El propósito de este trabajo, en consecuencia, consiste en analizar los aportes de la normativa contable a un mayor 
control de calidad del ejercicio profesional del Contador Público por la vía de la ética praxeológica.

De lo anterior se desprende que este trabajo comporta utilidad para los Contadores Públicos y para la sociedad en general, que se refleja en una óptima prestación de servicios, mayor responsabilidad social, riguroso cumplimiento de normas profesionales y postulados éticos, y, como consecuencia, mayor prestigio para la profesión.

\section{CONCLUSIONES}

El estudio de los contenidos en los dos Trabajos Interamericanos y de la Revista de CONTADURÍA PÚBLICA, incluyendo el material de trabajo usado en el Estudio de Investigación 2009, titulado "LA ÉTICA Y SU APORTE A LA CALIDAD QUE APLICA EL CONTADOR PÚBLICO EN EL PERÚ”, servirán de base para proponer la aprobación de nuevas bases para la preparación de un curso de ÉTICA Y DEONTOLOGÍA PROFESIONAL, en el avance de cambios en la formación del Contador Público, que necesariamente tiene que efectuar la Facultad de Ciencias Contables de la UNMSM, para actualizar el vigente Plan $\mathrm{Cu}$ rricular de Estudios,

El indicado curso de ÉTICA Y DEONTOLOGÍA PROFESIONAL, que deberá situarse en el área de especialidades del futuro Plan Curricular de Estudios, que se ubica del séptimo al décimo semestre académico; incluirá temáticas distintas en función de la Escuela Académico Profesional de Contabilidad, Escuela Académico Profesional de Gestión Tributaria y Escuela Académico
Profesional de Auditoría Empresarial y del Sector Público.

\section{RECOMENDACIONES}

Los contenidos completos de los dos trabajos interamericanos y de la Revista de CONTADURÍA PÚBLICA, que se muestran en el presente artículo, serán entregados al Instituto de Investigación de la Facultad de Ciencias Contables, recomendando su difusión a los docentes, para recibir posteriores aportaciones que se puedan agregar en el próximo trabajo de actualización del Plan Curricular de Estudios y colaborar en estructuración del Syllabus del nuevo curso de ÉTICA Y DEONTOLOGÍA PROFESIONAL.

En base al material técnico científico que se solicita su difusión, se recomienda a los docentes incluir en sus exposiciones académicas dirigidas a los estudiantes de Contabilidad, aquellos nuevos conceptos sobre Ética y Deontología Profesional que contribuirán a una mejor formación del Contador Público en el Perú.

\section{REFERENCIAS BIBLIOGRÁFICAS}

1. CONFERENCIA INTERAMERICANA DE CONTABILIDAD:

- Área 4. Comisión de Educación -"La Formación del Contador Público - Una propuesta para la Carrera de Grado”.

- Área 6. Comisión de Ética y Defensa Profesional - "De la Transdiciplinariedad de la Ética a los Códigos Deontológicos".

2. Revista CONTADURÍA PÚBLICA "Ética, Normativa y Calidad”. 